\title{
LAS BODEGAS TRADICIONALES: PATRIMONIO OLVIDADO EN LA CULTURA DEL VINO EN LA DENOMINACIÓN DE ORIGEN DE CIGALES (CASTILLA Y LEÓN, ESPAÑA)
}

\author{
J. Fernández Portela* \\ Departamento de Geografía de la Universidad de Valladolid \\ Facultad de Filosofía y Letras Plaza del Campus Universitario s/n C.P. 47011 Valladolid \\ jfportela@geo.uva.es / juliofernandezportela@hotmail.com \\ M.A. García Velasco \\ Instituto de Historia Simancas de la Universidad de Valladolid \\ Casa del Alcalde (Casa del Estudiante). Real de Burgos, s/n. C.P. 47011 Valladolid \\ magarciavelasco@gmail.com
}

\begin{abstract}
Resumen: Las bodegas tradicionales forman parte del patrimonio artístico y etnográfico existente en algunas localidades de Castilla y León. En el caso de los municipios que componen la Denominación de Origen (D.O.) de Cigales, este tipo de instalaciones ha adquirido un peso muy representativo debido a su abundancia con más de 1.200 bodegas repartidas por el territorio.

En la actualidad, estas bodegas han sufrido una serie de cambios muy importantes que están modificando el paisaje tradicional en el que se ubican. Si se pasea por los característicos barrios de bodegas de estos municipios se pueden observar todas las intervenciones realizadas por el hombre, y que en la mayoría de los casos no han seguido unos patrones comunes y acordes con el paisaje. Aspectos que no han contribuido más que a la pérdida de identidad de estos espacios, haciendo necesario plantear una serie de propuestas encaminadas a conservar estos barrios tan característicos.
\end{abstract}

Palabras clave: bodega tradicional, denominación de origen de Cigales, patrimonio, intervenciones.

Recibido: 22-09-13. Aceptado: 25-03-14.

* Investigador del Programa de Formación de Profesorado Universitario (FPU) del Ministerio de Educación del Gobierno de España. Miembro del proyecto Paisajes patrimoniales de la España interior septentrional y occidental (Ref. CSO2012 39564 C07 01). 


\begin{abstract}
Traditional cellars are part of the artistic and ethnographic heritage existing in some parts of Castilla y León. For municipalities that make up the Designation of Origin Cigales, these facilities have gained an important weight because of its abundance with more than 1,200 cellars scattered throughout the territory.

Currently, these cellars have suffered a series of major changes which are modifying the traditional landscape in which they are located. If you walk through the typical cellar neighbourhoods in these municipalities you can see all the interventions made by man, which in most cases have not followed a common pattern consistent with the landscape. Aspects that have contributed more to the loss of identity of these spaces, making it necessary to raise a number of proposals to keep these so characteristic neighbourhoods.
\end{abstract}

Key words: traditional cellar, designation of origin Cigales, heritage, interventions/actions.

\title{
1. Objetivos y metodología
}

El presente artículo tiene como objetivo analizar la situación actual existente en los barrios de bodegas de los municipios que componen la Denominación de Origen (D.O.) Cigales y proponer una serie de intervenciones que permitan ponerlos en valor planteando un conjunto de proyectos relacionados, principalmente, con el mundo del vino. Iniciativas que, a su vez, van a ayudar a mantener en un buen estado de conservación este rico patrimonio etnográfico tan característico de esta comarca. Estos espacios constituyen un recurso muy significativo que puede ayudar a lograr el desarrollo rural de estas localidades. El problema se encuentra en el estado de conservación de algunas de las bodegas que se han visto alteradas por las obras realizadas a posteriori por el hombre, desvirtuando su estructura original, por lo que es necesario intervenir en ellas lo antes posible para evitar una pérdida mayor de identidad.

Se plantea la realización de un diagnóstico y unas propuestas que permitan conocer los problemas existentes en los diferentes barrios de bodegas que componen el ámbito de estudio. Para ello, se ha realizado trabajo de campo de forma minuciosa por los once barrios, donde se ha podido comprobar la situación real existente en estos espacios, caracterizados por la presencia de un conjunto de problemas derivados del progresivo abandono de este patrimonio.

Se han realizado entrevistas a bodegueros y viticultores, principales agentes implicados en este sector, pero también han sido fundamentales los testimonios proporcio- 
nados por responsables políticos; empleados municipales (arquitectos, técnicos de cultura y de turismo), restauradores y hosteleros de la zona; así como los vecinos de estas localidades, sumando en total cincuenta entrevistas. Los perfiles seleccionados han sido una representación de los profesionales y personas más destacadas en los diversos ámbitos para que el resultado del estudio fuera lo más completo y enriquecedor posible. En estas entrevistas se han planteado una serie de ítems comunes, siendo los más representativos la percepción que tienen todos estos agentes sobre los barrios de bodegas, y si creen que tienen posibilidades de cara a futuras actuaciones que permitan dar un nuevo uso a estos espacios convirtiéndolos en un recurso socio-económico que contribuya a diversificar el tejido económico de los municipios.

Con toda la información recibida, se ha podido elaborar el diagnóstico de la situación actual existente en estos barrios, la elaboración de una matriz DAFO, y finalmente, se plantean una serie de intervenciones que pretenden poner en valor estos espacios y evitar una degradación mayor, además de proporcionar nuevas actividades económicas en estos municipios.

\section{El arraigo de la industria del vino en la comarca de Cigales}

El vino ha sido un producto que ha definido y delimitado la comarca de Cigales desde hace siglos elaborando los característicos claretes de estas tierras. Estos caldos han jugado un papel clave en la economía de los municipios que forman este espacio, ya que en el siglo $\mathrm{X}$ se elaboraban en Conforcos y en Covellas, actuales Corcos del Valle y Cubillas de Santa Marta respectivamente.

La importancia del clero y la nobleza ha sido clave para la expansión de los viñedos, y con ello de la elaboración de vino. La situación estratégica de esta comarca, en el centro de la actual Castilla y León, y concretamente, entre las ciudades de Valladolid y Palencia, lugar de paso para viajeros, comerciantes y trajineros entre el norte y el sur peninsular, permitió el asentamiento de colectivos sociales con fuerte poder adquisitivo, además de importantes consumidores del vino como eran los nobles y, en especial, los religiosos, que contribuyeron al resurgimiento de este cultivo tras la invasión de los musulmanes: "Todo el trabajo que supuso el hacer fructificar las tierras abandonadas no habría podido llevarse a cabo sin la organización y el fuerte impulso del clero" (Huetz de Lemps, 2005:129).

Este periodo de expansión se correspondió con el proceso de repoblación que tuvo lugar tras la expulsión de los musulmanes de las tierras castellanas, conocidas durante un tiempo como tierra de nadie por el abandono que sufrieron tras las luchas entre mu- 
sulmanes y cristianos por conquistar estos espacios ${ }^{1}$. Tras la reconquista se volvieron a ocupar los valles del Duero, y fueron las órdenes religiosas las que contribuyeron al asentamiento de la población a través del sistema de presura y la plantación de viñas a medias, permitiendo configurar una serie de centros productores vitivinícolas de gran relevancia como la Ribera del Duero, Rueda o Cigales, espacios que se corresponden con las Denominaciones de Origen más representativas de la región en la actualidad.

Desde entonces los viñedos comenzaron a ocupar los campos castellanos que hasta la fecha se caracterizaban por el cultivo, casi en exclusivo, de los cereales, haciendo honor al dicho de Castilla: granero de España. Se fue creando un paisaje más heterogéneo, combinando el trigo, la vid o los árboles frutales, que originaron un mosaico variado que describieron los viajeros europeos decimonónicos que atravesaron las llanuras castellanas, principalmente ingleses y franceses, en sus viajes a la Península Ibérica (Fernández Portela \& Isla García, 2012). Viajeros como Cock, Wünzer, Townsend, Twiss, Croker, Davillier o Gautier (Plasencia, 1995), plasmaron en sus memorias todo lo vivido durante su etapa en España. Especial interés mostraron hacia el cultivo de la vid y el consumo del vino, describiendo los viñedos que atravesaban o las bodegas que visitaban ${ }^{2}$.

Este periodo de expansión y de esplendor del viñedo en Cigales se vio alterado, al igual que en el resto de España, por la llegada de las plagas que asolaron las vides desde mediados del siglo XIX como el oídio, el mildéu, pero sobre todo la filoxera, con una difusión del parásito muy importante por prácticamente todo el territorio peninsular. Esta enfermedad redujo la totalidad de la superficie de viñedo existente en España en algunas provincias como Barcelona, Gerona, Málaga o Granada, y alrededor del 50\% en otras como sucedió en Valladolid, Zamora, La Rioja o Zaragoza (Solano Sobrado, 1991). En definitiva, una pérdida de más de 500.000 hectáreas (Piqueras Haba, 2005),

1. Las llanuras castellanas fueron denominadas tierra de nadie por algunos autores, ya que permanecieron como espacios destinados a la lucha entre musulmanes y cristianos quedando muchos territorios completamente despobladas. Como refleja Huetz de Lemps en la siguiente cita "Durante algunos siglos las llanuras altas del Duero pasan a ser una especie de tierra de nadie donde se pasean temibles ejércitos de musulmanes o cristianos que saquean, matan, y destruyen de forma sistemática ciudades, aldeas y cultivos" (Huetz de Lemps,2005:125). Idea que también recoge el historiador Julio Valdeón Baruque en su obra La Reconquista (2006) donde dice que “.. la Cuenca del Duero pasó a ser, a raíz de aquellas campañas, una zona intermedia entre al-Ándalus y el incipiente reino Astur" (56). Es decir, una tierra de nadie, un campo de batalla entre musulmanes y cristianos que arrasó con todo tipo de cultivos, casas, etc.

2. Numerosos fueron los viajeros que recorrieron estas tierras debido a su estratégica posición entre el sur y el norte peninsular. Las bodegas de la comarca de Cigales, y en concreto las de Dueñas llamaron la atención de muchos de ellos, entre los que se destacan a Teste que comentó lo siguiente "en la colina hay unos agujeros que se cierran con zarzas. Supuse que estos agujeros daban acceso a las bodegas...” (1959; 42). Más curioso fue el caso del francés Gautier, que se extrañaba de esta arquitectura tan diferente a la que había visto recientemente en las bodegas del sur de España. Gautier las describía como un tipo de instalaciones "cavadas en la roca viva, reciben la ventilación por torrecillas de forma de turbante, que ofrecen un falso aspecto de minarete muy singular" (recogido en Figueroa y Melgar, 1997; 247). 
lo que supuso un antes y un después en el cultivo de la vid y en la elaboración del vino, al introducir nuevas variedades resistentes a este parásito y nuevos métodos de producción procedentes de Francia.

La llegada del insecto a la cuenca del Duero se produjo a través del foco portugués de Gounvinhas, en el entorno de Oporto a finales del XIX, desde donde fue avanzando a través de las provincias de Zamora y Valladolid hasta llegar a la de Burgos, infectando los viñedos que encontraba a su paso, entre ellos los de los municipios que integran la comarca de Cigales. A la invasión natural de la filoxera hay que añadir la desidia y las negligencias de las autoridades nacionales, provinciales y locales en este asunto, y a las escasas iniciativas que se llevaron a cabo para solucionar el problema. Entre las más representativas se destaca el cordón sanitario en Cataluña propuesto por Juan Miret, o una iniciativa muy similar, conocida como Plan Logroño, principalmente en los viñedos del Ebro de las provincias de Logroño, Zaragoza, Navarra, Álava y Huesca, así como su ampliación a los territorios limítrofes de Burgos y Soria, pero que sin embargo, ambas, fueron iniciativas que nunca se pusieron en marcha (Camarero Bullón, 1989).

Desde entonces la superficie de viñedo de España, y en concreto la de Castilla y León, no se ha recuperado, y no ha vuelto a alcanzar las casi 300.000 hectáreas existentes a finales del siglo XIX. A esta enfermedad hay que sumar la crisis agraria de mediados del siglo XX que tuvo lugar en los campos castellanos a causa de diversas políticas como la implantación de los regadíos, la concentración parcelaria o la mecanización, que dieron lugar a un importante éxodo rural con la expulsión de población y la reducción de determinados cultivos, entre ellos los viñedos.

No fue hasta comienzos de la década de los ochenta del siglo XX cuando se produjo un resurgir de la industria vitivinícola en Castilla y León gracias a la aparición de las Denominaciones de Origen (Alonso Santos, et al., 2003), la primera de ellas la de Rueda en 1980 (Sánchez Hernández, 2002), la Ribera del Duero en 1982 (Aparicio et al., 2008), Toro en 1987 (Sánchez Hernández, 2003), el Bierzo en 1989 (Alonso Santos, 2003) y Cigales en 1991 (Fernández Portela, 2012) entre otras ${ }^{3}$. Esta figura de protección permitió la expansión del sector produciendo un vino de mayor calidad, y un mayor reconocimiento de los caldos elaborados en las bodegas de Castilla y León, no sólo en España, sino en todo el mundo.

En el caso de la D.O. Cigales, área de estudio de esta investigación, se compone de trece municipios que conforman este espacio, declarado como tal según la Orden

3. Hay que añadir las D.O. de Arlanza, Arribes, Tierra del Vino de Zamora y Tierra de León que obtuvieron la calificación en 2007; la Indicación Geográfica Protegida Vinos de la Tierra de Castilla y León; y los espacios con la mención de Vinos de Calidad de Valles de Benavente, Sierra de Salamanca y Valtiendas. 
de 9 de marzo de 1991 (Figura 1) ${ }^{4}$. Figura de calidad que viene a regular el sector vitivinícola de una comarca tradicional productora de vino, con una importante presencia de la vid en su territorio, así como la existencia de barrios de bodegas tradicionales que recuerdan la impronta histórica y económica que ha tenido desde siglos pasados esta actividad en los diferentes pueblos.

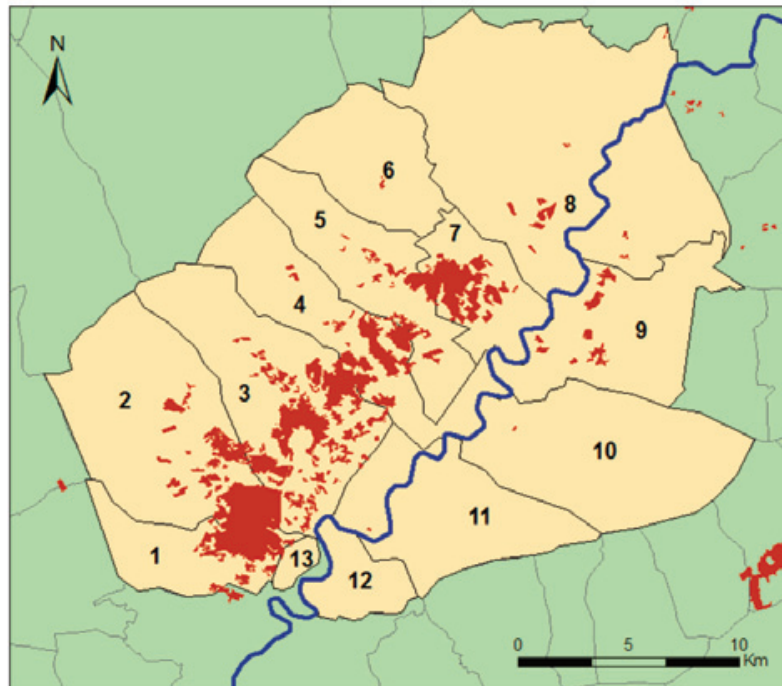

1. Fuensaldaña

2. Mucientes

3. Cigales

4. Corcos del Valle
5. Trigueros del Valle

6. Quintanilla de Trigueros

7. Cubillas de Santa Marta

8. Dueñas

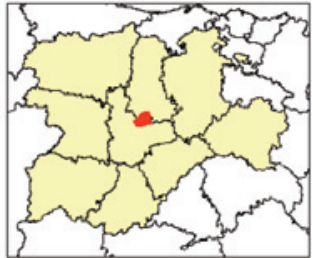

Castilla y León

D.O. Cigales

_ Rio Pisuerga/Pisuerga river

Viñedos/vineyards

D.O. Cigales

Otros municipios/

other municipalities

9. Valoria la Buena

10. San Martín de Valveni

11. Cabezón de Pisuerga

12. Santovenia de Pisuerga

13. Pago del Berrocal

Figura 1. Denominación de Origen de Cigales.

Fuente: Elaboración propia.

4. De la provincia de Valladolid son los siguientes: Cabezón de Pisuerga, Cigales, Corcos del Valle, Cubillas de Santa Marta, Fuensaldaña, Mucientes, Quintanilla de Trigueros, San Martín de Valvení, Santovenia de Pisuerga, Trigueros del Valle, Valoria la Buena, y el Pago del Berrocal en el municipio de Valladolid. De la provincia de Palencia: Dueñas. 


\section{Zarceras, respiraderos y cotarros: el paisaje de las bodegas tradicionales}

En 2011, la superficie de vides en esta D.O. era de 2.217,18 hectáreas, sin embargo, el 98\% de la superficie cultivada se localiza en la margen derecha del Pisuerga en municipios como Cigales, Cubillas de Santa Marta, Mucientes o Corcos del Valle, al igual que sucede con las nuevas bodegas industriales, de las cuales, 33 de las 34 existentes, se ubican también en la margen derecha del Pisuerga, aspectos que indican la mayor impronta que adquiere este cultivo y la elaboración de vino en estos municipios, siendo, históricamente, los más representativos.

El peso que ha tenido este cultivo a lo largo de la historia hizo necesaria la construcción de lagares para elaborar el vino y de bodegas para almacenarlo. El tipo característico de bodega tradicional de esta comarca es la subterránea localizada en las afueras de los municipios conformando barrios propios, algunos anexos a la trama urbana, otros separados por tierras de cultivos conformado una especie de "barrios islas" de diversas formas, mientras que otros han quedado inmersos dentro de la trama urbana como consecuencia del crecimiento que han experimentado algunas localidades.

Como elementos más representativos se encuentran la zarcera, una especie de ventana por la que se arroja la uva hacia el interior de la bodega; el respiradero, una chimenea compuesta por agujeros con el objetivo de ventilar las instalaciones, sobre todo en el periodo de fermentación; y los cotarros, montículos de tierra extraída del interior de las bodegas durante su construcción, y que se depositan en la parte superior de las mismas con el objetivo de ejercer un efecto aislante. Junto a estos elementos hay que añadir otros como las propias chimeneas, diferentes a los respiraderos, las características fachadas de piedra o los poyos de sus puertas.

En los municipios que conforman la D.O. Cigales, este tipo de construcciones son muy características y abundantes. Existen un total de 1.200 bodegas catalogadas y 47 lagares (Cuadro 1) distribuidos de forma desigual por el territorio.

La importancia y riqueza que posee este patrimonio en el territorio ha suscitado cierto interés para su conservación. Son barrios de bodegas muy característicos que constituyen un reclamo importante para los turistas ávidos de conocer algo más sobre el mundo del vino. Son un recurso patrimonial clave para el desarrollo rural de estas localidades, y constituyen un complemento de la oferta enoturística de la comarca junto a la visita de las bodegas industriales, la fiesta de la Vendimia de Cigales, el Aula de interpretación del vino de Mucientes, o del Museo del Cántaro de Valoria la Buena entre otras actividades y eventos que se organizan en torno al mundo del vino.

A pesar de todo, estos barrios presentan una serie de problemas que habría que solucionar para evitar que pierdan la identidad propia que poseen. En los últimos años algunos de ellos se han ido desvirtuando al aparecer nuevas construcciones que rea- 
lizan la función de merenderos, y que rompen por completo con la estética tan característica de estos espacios. También se han producido obras de reforma en las fachadas y en algunos de los elementos más característicos de las bodegas como son los respiraderos, las zarceras o las chimeneas, modificando de forma drástica el típico paisaje alomado que forman este tipo de construcciones.

Cuadro 1. Bodegas tradicionales y lagares en los municipios de la D.O. Cigales

\begin{tabular}{|l|c|c|}
\hline Municipio & Bodegas & Lagares \\
\hline Cabezón & 68 & 0 \\
Cigales & 155 & 17 \\
Corcos & 82 & 12 \\
Cubillas & 77 & 7 \\
Dueñas & 228 & 1 \\
Fuensaldaña & 92 & 0 \\
Mucientes & 151 & 0 \\
Quintanilla & 28 & 0 \\
San Martín & 44 & 1 \\
Santovenia & 0 & 0 \\
Trigueros & 130 & 9 \\
Valoria & 145 & 0 \\
El Berrocal & 0 & 0 \\
\hline Total & 1200 & 47 \\
\hline
\end{tabular}

Fuente: Fichas de Catalogación de Bodegas, Lagares y Guardaviñas, proporcionadas por la Consejería de Cultura y Turismo de la Junta de Castilla y León (2007). Elaboración propia.

\section{Marco normativo y diagnóstico de los principales problemas en los barrios de bodegas}

El siguiente diagnóstico, realizado a través del trabajo de campo llevado a cabo en los once barrios de bodegas existentes ${ }^{5}$, pretende mostrar todos los factores, tanto positivos como negativos, de las bodegas tradicionales con la finalidad de poder llevar a cabo una serie de intervenciones, siempre desde la preservación del patrimonio cultural, que mejoren estos espacios.

5. De los municipios existentes en la D.O. Cigales, todos poseen barrio de bodegas a excepción de Santovenia de Pisuerga y el Pago del Berrocal. 
La característica principal de las bodegas tradicionales es su ubicación en barrios propios en las afueras de los núcleos urbanos conformando un paisaje peculiar de cotarros. Este patrimonio vernáculo está vinculado al trabajo de los antepasados y con varias centurias de esfuerzo y de historia, pero que, no viene reflejado en la mayoría de los casos con un respeto y cuidado de estos espacios. Estos problemas también se derivan de la escasa materia legislativa existente para este tipo de construcciones que se encuentran desamparadas en la mayoría de las ocasiones.

El marco normativo que debe amparar a las bodegas es algo complejo, ya que es necesario integrar el patrimonio, el urbanismo, el medio ambiente y la economía. Las leyes estatales trazan las líneas generales, pero actualmente quienes tienen las competencias en estas áreas son las Comunidades Autónomas, por lo que se rige por la legislación de Castilla y León.

La normativa regional sobre patrimonio carece de menciones específicas sobre paisaje, sin embargo sí que aparece en la Ley de Urbanismo de Castilla y León 5/1999 de 8 de abril. En su artículo 9 se refleja que el uso del suelo y, en especial, su urbanización y edificación deberá adaptarse a las características naturales y culturales de su ambiente. En áreas de manifiesto valor natural o cultural, en especial en el interior o en el entorno de los Espacios Naturales Protegidos y de los inmuebles declarados como Bien de Interés Cultural, no se permitirá que las construcciones e instalaciones de nueva planta, así como la reforma, rehabilitación o ampliación de las existentes, y las instalaciones de suministro de servicios, degraden la armonía del paisaje o impidan la contemplación del mismo. A tal efecto, se exigirá que todas ellas armonicen con su entorno inmediato y con el paisaje circundante en cuanto a su situación, uso, altura, volumen color, composición, materiales y demás características, tanto propias como de sus elementos complementarios.

Esta ley se desarrolla en el Reglamento de Urbanismo de Castilla y León, Decreto 22/2044, de 29 de enero, en las Directrices Esenciales de Ordenación del Territorio de Castilla y León (Ley 3/2008 de 17 de junio), donde entre los artículos 3 y 10, se mencionan los paisajes culturales, así como en las Medidas sobre Urbanismo y Suelo de la Ley $4 / 2008$ de 15 de septiembre en su artículo $4^{\circ}$.

Si a nivel estatal, existe la Ley de Patrimonio Histórico de 1985, con la creación de las Comunidades Autónomas y el traslado de las competencias, se promulgó la Ley de Patrimonio Cultural de Castilla y León 12/2002. En su artículo 42.2 dedicado a la Conservación de conjuntos históricos, sitios históricos, zonas arqueológicas y conjuntos etnológicos, se recoge lo siguiente "La conservación de los conjuntos históricos comporta el mantenimiento de la estructura urbana y arquitectónica y de la silueta paisajística, así como de las características generales de su ambiente. Se considerarán excepcionales las sustituciones de inmuebles y sólo podrán realizarse en la medida que contribuyan a la conservación general del carácter del conjunto. La conservación de los sitios his- 
tóricos y conjuntos etnológicos comporta el mantenimiento de los valores históricos, etnológicos, paleontológicos y antropológicos, el paisaje y las características generales de su ambiente". Esto produciría que en "los sitios históricos y zonas arqueológicas queda prohibida la colocación de cualquier clase de publicidad, así como cables, antenas y conducciones aparentes. Sólo en el caso en que se sitúen sobre suelo urbano se podrán autorizar dichas instalaciones, siempre que guarden armonía con el ambiente en el que se encuentren".

Dentro de las comunidades autónomas es importante la regulación que se realiza en cuanto a la ordenación del territorio y el desarrollo de las leyes. La legislación en materia de suelo es importante para darle un tipo de uso acorde al desarrollo de este patrimonio. No existe una normativa concreta enfocada a los barrios de bodegas. Las actuaciones que hay que realizar son complejas al abarcar varias áreas y en muchas ocasiones el condicionante económico frena los estudios y proyectos a desarrollar. Cada vez se están derrumbando más bodegas y se deja al criterio de los propietarios la restauración de las mismas, aunque, es cierto que, en algunos ayuntamientos se están llevando a cabo una serie de iniciativas para proteger estos lugares. Según la sensibilidad de los ayuntamientos se regula con mayor o menor rigurosidad. Como ejemplos positivos se encuentran los casos del municipio de Cigales, que en sus Normas Urbanísticas, y en concreto en el Artículo 46, aparece la Ordenanza de las zonas de bodegas (BO), o en el caso de Mucientes que se las considera como Usos en suelos rústico de especial protección (SREP), donde se recogen una serie de aspectos a tener en cuenta para preservar y conservar este tipo de instalaciones ${ }^{6}$.

El incumplimiento de la escasa legislación existente hacia este patrimonio, ha dado lugar a una serie de importantes problemas en los barrios de bodegas de los municipios que componen la D.O. Cigales (Figura 2).

A continuación se detalla un listado con diez problemas observados en estos espacios:

1. Mala accesibilidad al interior de las bodegas. El acceso es a través de unas escaleras muy empinadas, y no es posible la instalación de ascensores ni de rampas para personas con movilidad reducida. Las calles de los barrios suelen ser estrechas y se cruzan entre ellas dando lugar a laberintos donde es fácil perderse.

6. El ejemplo más significativo es el acaecido en Aranda de Duero, donde la Consejería de Cultura y Turismo de la Junta de Castilla y León, ha comenzado los trámites para declarar como Bien de Interés Cultural (BIC), dentro de los conjuntos etnológicos, al barrio de bodegas de la localidad burgalesa de Aranda de Duero (BOCYL, Martes 11 de junio de 2013). En una línea de trabajo similar se encuentran otros municipios que tuvieron una impronta vitivinícola en el pasado como las localidades palentinas de Torquemada y Baltanás con el objetivo de evitar que estas instalaciones se conviertan en un montón de tierra, propuestas que, de momento, no han conseguido el objetivo esperado. 


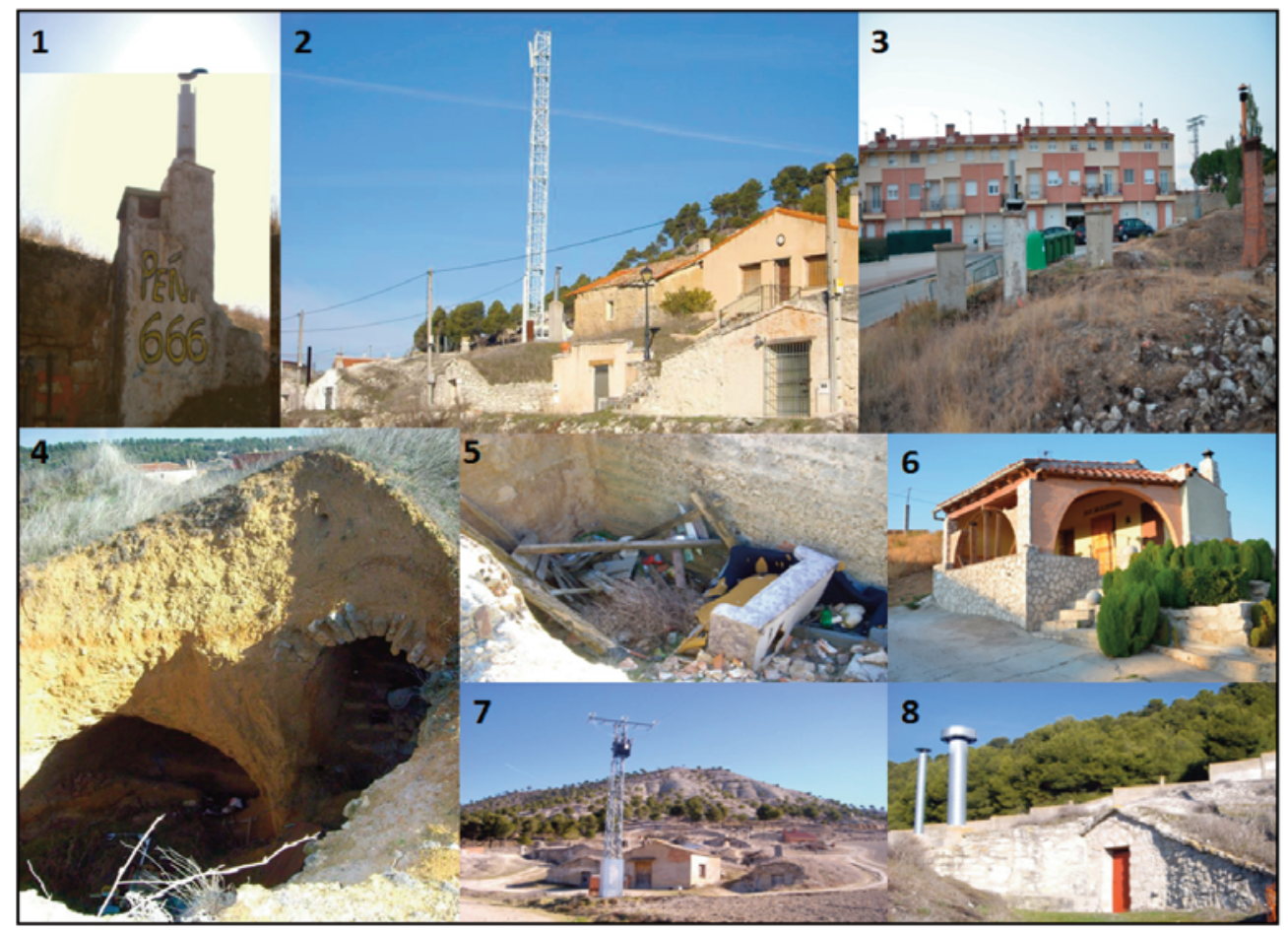

Figura 2. Problemas en los barrios de bodegas tradicionales de la D.O. Cigales. 1) Cabezón de Pisuerga. 2) Corcos del Valle. 3) Cigales. 4) Cubillas de Santa Marta. 5) Mucientes. 6) Fuensaldaña. 7) San Martín de Valvení. 8) Corcos del Valle.

Fuente: Fotografías de los autores.

2. Títulos de propiedad. A nivel administrativo esto representa un problema importante. Al ser construcciones de hace siglos apenas existe documentación de su construcción, de quién la hizo, etc., es una arquitectura sin arquitectos (Franco \& Luis, 2005). A esto hay que sumar la importante fragmentación existente en la propiedad como consecuencia de las herencias. En muchos casos las propiedades se encuentran sin legalizar, y en otras ocasiones hay decenas de propietarios que no se ponen de acuerdo a la hora de invertir dinero en su mantenimiento y se acaba abandonando la bodega hasta su hundimiento.

3. Derrumbe. Algunas de las bodegas se encuentran hundidas, y los agujeros existentes como consecuencia del hundimiento del techo son aprovechados para tirar basuras y escombros. También es peligroso para las bodegas colindantes que pueden ver afectadas sus estructuras. 
4. Cableado y postes eléctricos en las fachadas. La llegada de la luz a estos barrios ha supuesto la instalación de postes eléctricos y cableado para suministrar electricidad a estas construcciones, así como las cajas de la luz que, en la mayoría de los casos, se han ubicado en las fachadas de las bodegas junto a las puertas, y que no se ha hecho nada por intentar camuflarlas o adaptarlas lo máximo posible al paisaje tradicional. A estos cables hay que añadir la proliferación de antenas de telefonía móvil aprovechando los cerros o pequeñas lomas para su ubicación.

5. Falta de unidad estética en fachadas y otros elementos arquitectónicos exteriores. El patrimonio etnológico se encuentra, fundamentalmente, en el interior de las bodegas. Sin embargo, las fachadas, las chimeneas, las zarceras y los respiraderos son la cara al exterior de las bodegas, y estos elementos arquitectónicos se han ido modificando con el tiempo. Al no existir una legislación específica que regule cómo deben hacerse estas reformas, han surgido elementos con materiales diversos que no respetan la estética tradicional.

6. Escombros en las puertas. El escaso interés por parte de determinados propietarios de lo que supone poseer una de estas bodegas da lugar a una escasa preocupación por las mismas y por su entorno. Cuando tienen que realizar obras, es habitual deshacerse de los escombros en las inmediaciones de la misma.

7. Zonas de peñas y de botellón. Las bodegas tradicionales se caracterizan, a día de hoy, por la celebración de eventos sociales más que por la elaboración de vino, por lo que se han convertido en espacios de ocio y esparcimiento de la población, y entre ella de los más jóvenes. Al ser zonas apartadas son lugares muy propios para la realización de estas actividades donde se concentran restos de basura y de desperdicios, y la presencia de pintadas o grafitis en las fachadas o las chimeneas son habituales ante la falta de sensibilidad de los jóvenes hacia estas construcciones. A esta suciedad hay que sumar el crecimiento de maleza en los cotarros y caminos donde se pueden producir incendios en los meses estivales.

8. Construcción de naves o añadidos que distorsionan la bodega tradicional. En la última década ha sido muy habitual la construcción de merenderos en los barrios de bodegas con todo tipo de comodidades, sin respetar la estética, y desvirtuando el paisaje tradicional.

9. Presencia de perros sueltos. El mundo rural no debe ser sinónimo de retraso o de falta de normas. Los perros son frecuentes para proteger las propiedades, chalets o naves agrarias, y en muchas ocasiones se escapan y vagan libres por estos barrios, lo que provoca el miedo entre los visitantes.

10. Falta de señalización. En la mayoría de los casos no hay un acceso al barrio de las bodegas, por lo que no consideran apropiado darlo a conocer al visitante. 
Partimos de estas premisas negativas como punto de partida a partir de las cuáles se debe actuar para mejorar la situación de estos espacios. Para ello es necesaria la ayuda de diferentes agentes sociales y económicos que permitan lograr unos objetivos que posibiliten el desarrollo de estos lugares y darles un nuevo uso turístico diferente al original que potencie los recursos endógenos de los diversos pueblos, contribuyendo a diversificar su economía, basada, en la mayor parte de ellos, en la agricultura.

Teniendo en cuenta este diagnóstico, se ha elaborado una matriz D.A.F.O. donde se reflejan las Debilidades, las Amenazas, las Fortalezas y las Oportunidades de los barrios de bodegas y del entorno de los mismos (Cuadro 2). Esta matriz permite profundizar en los puntos fuertes y débiles de este territorio, aspectos que van a permitir definir una serie de intervenciones encaminadas a recuperar estos barrios a través de propuestas que pongan en valor el territorio y utilicen este tipo de construcciones.

\section{Propuesta de intervención en los barrios de bodegas tradicionales}

A esta economía propia del cultivo de la vid y la producción del vino se le pueden unir diversas posibilidades de poner en valor el conjunto, y de organizar diversos programas culturales, sociales o empresariales, en las que se pueda comunicar la historia, cultura y las tradiciones del lugar con el eje vertebrador de las bodegas.

La mejor manera de preservar el Patrimonio es dotándolo de usos y, por tanto, haciendo rentables las necesarias inversiones para su restauración y rehabilitación. El abandono derivado del desuso es posible revertirlo con la rehabilitación para nuevas dedicaciones. Es una realidad que se puede apreciar en las bodegas que se encuentran en mejor estado de conservación, y que no sería descartable contemplar la posibilidad futura de rehabilitaciones integrales de envergadura que permitan recibir nuevos usos ligados a las demandas del presente. Se trata de utilizarlo como recurso para preservar este patrimonio histórico-etnográfico (Marino Pascual, 2010).

Con el análisis realizado, podemos trazar diversas líneas de actuación para realizar en los barrios de bodegas, dándoles unos usos diferentes a los que tuvieron una vez y a los presentes. En la actualidad, salvo la actividad eminentemente social que tienen las bodegas, hay escasos ejemplos de otros usos como pueden ser los destinados, principalmente, a la restauración. A continuación se presenta una propuesta formada por diversas iniciativas que puedan albergar actividades que giren en torno al mundo del vino, a la vez que permitan potenciar el tejido económico y empresarial del conjunto de estos municipios. Esta propuesta estaría formada por un ecomuseo, un museo al aire libre, un festival de bodegas tradicionales, sala de exposiciones, vivero de empresas creativo-culturales, bodegas de creación, restaurante cultural, alojamientos rurales culturales y un centro de degustación enogastronómica. A continuación se detalla cada una de estas posibles intervenciones: 


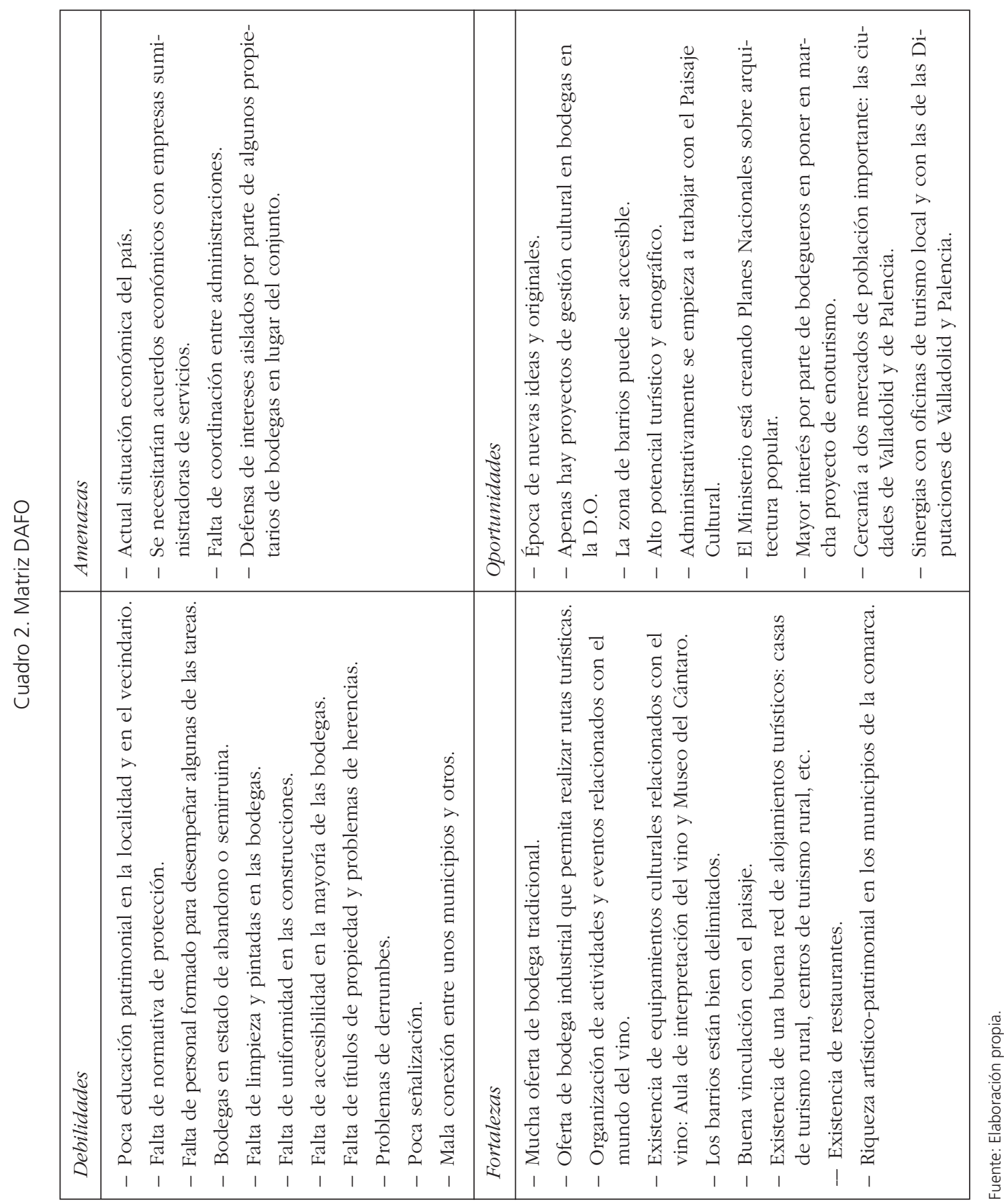




\subsection{Apertura de un Ecomuseo}

Según el Comité de Historia Natural del ICOM, el ecomuseo ${ }^{7}$ es una centro museístico que gestiona, estudia y valora con finalidades científicas, educativas y, en general, culturales, el patrimonio general de una comunidad específica, incluido el ambiente natural y cultural del medio. De este modo, el ecomuseo es un vehículo para la participación cívica en la proyección y en el desarrollo colectivo. Con este objetivo se sirve de todos los instrumentos y los métodos a su disposición con el fin de permitir al público comprender, juzgar y gestionar, de forma responsable y libre, los problemas con los que debe enfrentarse. El ecomuseo utiliza una estructura popular, la realidad de la vida cotidiana y de las situaciones concretas para alcanzar los cambios deseados y lograr el bienestar y del desarrollo de la comunidad.

Dentro de la Campiña del Pisuerga, el ecomuseo daría a conocer cómo el ser humano ha aprovechado los recursos que le ofrece la naturaleza (tierra, agua, plantas, animales... $)^{8}$ para valorar la importancia de vivir en armonía con todos estos elementos en relación a las bodegas y su evolución socioeconómica, ya que la vinculación entre el hombre, los animales y la naturaleza ha sido una constante a lo largo de la historia.

El proyecto de ecomuseo 9 nace con la intención de recuperar, mantener, profundizar y dinamizar los valores que constituyen la identidad tradicional de esa zona. La idea que se pretende es aglutinar en él, todos aquellos usos, costumbres y tradiciones, vinculados al entorno en el que han sido desarrollados, en este caso, la cultura del vino en la zona y más en concreto, en la localidad donde se asiente el ecomuso.

Se recuperaría una bodega tradicional realizando un recorrido museístico en la zona interior visitando el lagar, las sisas, la cocina, etc., dedicando un espacio importante a la figura del bodeguero. El ecomuseo debe reflejar la siguiente inquietud que tiene el visitante: ¿Cómo vivían y trabajaban los habitantes de estas localidades antes de la llegada de la industrialización?

También puede recoger la vinculación de las bodegas con la ganadería como forma más sostenible de mantener el paisaje, y el correcto mantenimiento de los cotarros.

7. Los objetivos del Ecomuseo se pueden ver en www.ecomuseoitinerante.com/proyecto/objetivos.html

8. Un ejemplo relativamente cercano lo tenemos en Ampudia, Palencia, en La Huerta de Valoria (www.lahuertadevaloria.com), en el que se trabaja con una bodega, dando un resultado positivo. Se debe aprovechar las potencialidades que presenta la zona de estudio en esta materia, ya que por el Bajo Valle del Pisuerga transcurre el río Pisuerga que cuenta en su ribera con una Zona de Especial Protección para las Aves (ZEPA) y un Lugar de Interés Comunitario (LIC), además de la existencia del Canal de Castilla.

9. A las afueras de Ampudia existe un Ecomuseo donde una de las partes es la visita a una bodega, totalmente adecentada, realizando diversas actividades que vinculen la educación con el patrimonio y la naturaleza. 
La propuesta también puede suponer el desarrollo de un modelo para la puesta en marcha de itinerarios temáticos, con la visita y desarrollo de actividades en los barrios de bodegas, donde confluyen elementos patrimoniales significativos relacionados con el vino. Igualmente, y como elemento motivador y museográfico de apoyo, creando estructuras expositivas itinerantes que ilustren la temática.

\subsection{Museo de Arte al Aire Libre}

Esta propuesta debe incidir sobre las bodegas, los senderos y viales principales. La creación de este espacio se realiza como forma de acercar el arte al ciudadano de a pie para que tenga más sensibilidad aprovechando las posibilidades que nos ofrece este entorno al aire libre.

Los barrios de bodegas son propicios para hacer un Museo de Arte al Aire Libre, vinculado a la sostenibilidad, la cultura y la naturaleza de forma que se haga atractiva tanto para el vecindario como para los visitantes. Elementos como la fachada o el dintel, el respiradero o las zarceras, son lugares perfectamente utilizables para ser pintados con diferentes variedades artísticas, lo que permitiría camuflar algunas de las nuevas construcciones que se han realizado y que rompen con la estética del paisaje, por lo que el impacto visual sería menor ${ }^{10}$.

Actualmente, existe disparidad de materiales en la elaboración de estas partes: ladrillo, mampostería, piedra, pvc o cemento entre un largo listado. Para embellecerlo y darle un cariz apropiado al patrimonio existente, se debería unificar el criterio según el material tradicional de la zona. Sin embargo, aunque es recomendable, no es óbice para poder pintar estas partes y realizar un Museo de Arte al Aire Libre. Cierto estilo podemos encontrar en el Barrio de bodegas de la localidad riojana de Haro, municipio donde se ubican diversas esculturas relacionadas con el vino por el barrio de bodegas, pero sobre todo paredes "medianiles" con motivos de la industria del vino que cubren los muros de cemento que restaban valor al conjunto ${ }^{11}$.

También se puede utilizar la escultura, bien como complemento, o bien como forma artística del Museo. Para ello es recomendable que los materiales sean ligeros pero duraderos debido a la inestabilidad del terreno.

Otro aspecto sociológico de esta propuesta se encuentra en que si los propios jóvenes se identifican con parte del arte que aparece, o bien alguno de ellos sea el propio artista, seguramente, respeten más esta zona y lo consideren como algo suyo para no destruir.

10. Una propuesta similar se está realizando en el entorno natural de la Sierra de Huelva como se puede ver en www.sierracentrodearte.com, así como en un sector de las bodegas de Valdevimbre en la D.O. Tierra de León, donde han pintado todas las chimeneas en tonos ocres para esconder las capas de cemento.

11. http://www.haroturismo.org/es/actuaciones/index 


\subsection{Festival de Bodegas tradicionales}

Este festival puede ser un evento cultural con mucho recorrido según el grado de participación de los propietarios, el apoyo de las administraciones, patrocinadores, asociaciones, etc. Puede llegar tan lejos como estime la sociedad vinculada al territorio. Para ello, es importante marcar de forma clara los objetivos para realizar un Festival como, por ejemplo, el interés de los propietarios de bodegas en adecentar, embellecer y arreglar su patrimonio para el deleite y disfrute tanto propio como de los visitantes. Se debe crear un sano sentimiento de competitividad que produzca un beneficio común en la puesta a punto de las bodegas.

Este festival puede y debe ser también una fuente para atraer turismo y riqueza económica al municipio con esta fiesta popular. Es por ello importante, que dentro de la programación existan actividades que propicien esta búsqueda de recursos económicos. Este evento debe encuadrarse bajo un certamen anual conocido en el calendario, y en cuya celebración se involucre la mayor parte de la localidad, dándole una promoción necesaria para la asistencia de visitantes, como sucede en el Festival de Patios que se realiza anualmente en Córdoba desde 1918, siendo uno de los referentes culturales y turísticos de Córdoba ${ }^{12}$.

Los propietarios tendrían que enseñar su bodega de forma gratuita durante un periodo de tiempo determinado en un horario establecido. Existirían diversos premios con varias categorías como por ejemplo la bodega mejor conservada o el interior mejor adaptado a lo que era una bodega tradicional

El jurado puede estar formado por profesionales, por personas vinculadas a la gestión cultural, al patrimonio, o bien por el voto de los visitantes mediante unas urnas ubicadas en un punto localizado. A medida que la actividad vaya creciendo en bodegas participantes y público asistente, se debe complementar con actividades que involucren al resto del pueblo, fundamentalmente con las asociaciones y empresarios existentes en el municipio, con actuaciones musicales o bailes regionales.

Las actividades se pueden complementar con un programa educativo para dar a conocer a los estudiantes, la arquitectura y el patrimonio, así como promover el respeto por el mismo. Este programa se puede realizar con el alumnado de los colegios, institutos y/o bien con alumnos universitarios interesados en este mundo como pueden ser los de arquitectura, enología, economía, geografía, historia o historia del arte.

12. Información obtenida en www.mayocordobes.es/patios.php 


\subsection{Sala de Exposiciones}

Muchas localidades no disponen de Sala de Exposiciones y es una de las demandas que la sociedad vinculada a la cultura está realizando, especialmente en el medio rural. No por vivir alejados de las urbes se tiene que estar de espaldas al conocimiento del arte.

Esta propuesta tiene que pulir algunos aspectos puesto que la bodega debe estar en un lugar de fácil acceso y señalización. Al estar las bodegas en lugares un tanto alejados en el extrarradio de la localidad, la sociedad lo ve lejano y es necesario trabajar con campañas promocionales para que la ciudadanía se acerque a la bodega para contemplar las exposiciones.

También es importante encontrar una bodega que esté en buenas condiciones de exposición, con un recorrido amplio, posibilidad de salida de emergencia, de incluir servicios públicos, así como tener en cuenta el tema de accesibilidad. Estos apartados, según se encuentre la normativa vigente, pero sería ideal poder contemplarlo.

Hay una experiencia en Murcia ${ }^{13}$ donde utilizan una antigua bodega de aceite como centro de exposiciones. Para la propia Fundación Casa Pintada, la experiencia es muy positiva ya que los artistas están deseando exponer en un lugar totalmente diferente, original e innovador.

\subsection{Vivero de empresas creativo-culturales}

La ubicación tan cercana a Valladolid de algunas localidades, propicia que sea factible la propuesta de crear un vivero de empresas culturales en las bodegas tradicionales. El sector creativo-cultural es un sector que actualmente está en auge. Gracias a las nuevas tecnologías, no es necesario trabajar en el centro de las ciudades sino que también se pueden utilizar enclaves que ofrezcan otras ventajas para los emprendedores. En estas bodegas se pueden desarrollar su futuro para crear una fuente de empleo y de creación de riqueza.

En la actualidad hay diversos proyectos vinculados a las ciudades y al uso de instalaciones industriales. La cercanía a la urbe y el apoyo básico en las nuevas tecnologías, propicia que sea factible este tipo de empresas en el medio rural, siempre y cuando se cumplan unas condiciones mínimas en este vivero.

13. Ubicado en La Casa Pintada, de Mula (Murcia) y gestionado por la Fundación Casa-Pintada. Lo utilizan como lugar expositivo de exposiciones temporales. Se puede obtener más información en www.casapintada.gabarron.org/LaCasaPintada.aspx La buena valoración de esta iniciativa procede de la información suministrada por las fuentes internas de esta Fundación. 
Sin embargo, al ser una iniciativa empresarial, requiere unos acuerdos administrativos y ajustarse especialmente a la normativa en cuanto a seguridad, accesibilidad, etc. Hay que estudiar, investigar y analizar las bodegas que pueden concurrir a ello y en otros casos, coordinarse entre las administraciones para facilitar esta propuesta y hacerla acorde con la normativa.

Dentro del grupo de bodegas existentes se pueden escoger unas 8-10 para poder realizar este vivero. Cada una de forma independiente aunque los servicios se puedan poner en común. Cada bodega estaría alquilada a un bajo coste para los emprendedores, y en este coste de alquiler estaría incluido el mantenimiento, los servicios de luz, agua, mobiliario básico y conexión a internet. El abastecimiento habría que analizarlo según las posibilidades, para hacerlas de forma individual en cada bodega, o bien mancomunada entre todas ellas.

Los empleos culturales a los que van dirigidos son diseñadores gráficos, artesanos, artistas, grupos de teatro o incluso asociaciones que puedan tener un local para trabajar sobre sus fines. La administración debe coordinar esta propuesta junto con otros entes administrativos superiores y los propietarios de las bodegas.

\subsection{Bodegas de creación}

La propuesta es compleja pero muy atractiva puesto que dinamizaría las localidades desde el punto de vista del desarrollo artístico y la industria cultural.

El objetivo es dotar a las bodegas como centros de creación artística y cultural bajo la marca "Bodegas de creación"14. Para ello habría que seleccionar qué bodegas se ajustan más a las necesidades, puesto que no todas valen por el tamaño o situación, además de la propiedad existente que en muchos de los casos habría que negociar. Es fundamental la coordinación entre administraciones puesto que el proyecto es ambicioso y lo suficientemente atractivo como para implicarse todas las partes posibles.

El programa Bodegas de Creación se basa en una línea de actuaciones que pongan la creatividad en un primer plano, a través de la creación de espacios, pero sobre todo de redes de conexión entre colectivos y artistas individuales. La propuesta es la reutilización de bodegas existentes para convertirlos en lugares de creación, donde la gestión se haga de forma compartida entre la Administración Pública y los propios artistas. La idea es que los presupuestos vayan fundamentalmente a la creación de programas formativos, asesoramiento y recursos técnicos.

14. Siguiendo el modelo de Fábricas de creación existente en Barcelona y Euskadi con el uso de naves industriales que se encontraban totalmente abandonadas. 
Además de esta actuación, el plan incluye la creación de una red de experiencias creativas, destinada a colectivos de producción artística, con la intención de crear vías de intercambio de información entre personas relacionadas con la creación cultural dentro de un marco estable. En este sentido, algunas de las propuestas son la puesta en marcha de planes de formación (no en el ámbito artístico sino en el del asesoramiento para proyectos relacionados con la industria cultural), la creación de una oficina coordinadora para canalizar recursos y promover proyectos comunes, y la organización de jornadas y encuentros anuales ${ }^{15}$.

La parte negativa estriba, además de en la accesibilidad, en las dimensiones de la bodega, lo que limitaría el arte que se puede trabajar dentro de la misma. Sin embargo, conociendo las limitaciones y enfocando claramente el tipo de arte con el que se puede trabajar, el proyecto puede salir adelante. Los proyectos vinculados a las Bodegas de Creación deben compartir un hilo argumental como es el respeto y la valorización del legado patrimonial popular castellano y su adaptación a los nuevos tiempos y usuarios.

\subsection{Restaurante cultural}

En la actualidad existen varios restaurantes ubicados sobre bodegas privadas. Algunos han respetado el paisaje y el entorno mejor que otros. Lo que les diferencia es la ubicación, la decoración o el toque patrimonial, aspectos que no pueden ofrecer otro tipo de restaurantes, y que sólo los que se encuentran en las bodegas de estas localidades lo pueden hacer. Estos centros pueden disponer de lugares expositivos dentro de las salas para que los artistas puedan mostrar sus obras de arte. Se utilizaría el restaurante con una finalidad cultural, tanto en lo puramente decorativo, como de promoción de los artistas o comercialización de sus obras.

La parte más complicada se establece en cumplir la normativa vigente en materia de sanidad, seguridad, industria y accesibilidad a discapacitados. Al poder recibir personas se necesita diversos requisitos que no todas las bodegas pueden asumir por su tipología. Es por ello que es necesario comprobar qué bodegas se pueden ceñir a los criterios de seguridad (salidas de emergencia), incendios y accesibilidad.

Una propuesta aconsejable para el restaurante es que con carácter regular pueda realizar actividades complementarias en una parte del mismo como monólogos o conciertos de música de pocos decibelios, ya que un valor grande de los mismos podría dañar las instalaciones.

15. Obtenido de www.euskadinnova.net También se puede ver la organización de estos centros en www.bcn.cat/fabriquesdecreacio/ca/ 


\subsection{Alojamiento rural cultural}

Una propuesta innovadora, con iniciativa privada, pero compleja en cuanto a la realización por la legislación actual, es la creación de un hotel con encanto en las bodegas. Tenemos referencias de actuaciones realizadas en la localidad italiana de Matera, donde se ha dado uso a casas-cuevas y cuevas prehistóricas convirtiéndolas en hoteles de lujo.

La Ley 14/2010, de 9 de diciembre, de Turismo de Castilla y León nos ofrece dos tipos de Alojamiento que se pueden ajustar a la propuesta: "Hotel con historia" es el establecimiento situado en un inmueble que cumpla alguno de los siguientes requisitos: haber sido declarado bien de interés cultural o inventariado de acuerdo con lo establecido en la normativa sobre patrimonio cultural; estar integrado en un Conjunto Histórico, siempre que ostente los valores que determinaron su declaración como bien de interés cultural o haber sido escenario de un acontecimiento histórico relevante. "Posada" entendiendo por tal el establecimiento ubicado en un edificio con valor arquitectónico tradicional, histórico, cultural o etnográfico.

Analizada las posibilidades que se pueden dar con el tipo de alojamiento y la realidad existente, en primer lugar, hay que decidir el entorno de bodegas exactas que se quiere realizar. Este alojamiento puede complementarse con salas de exposición para que los artistas puedan exhibir sus cuadros. Se utilizaría el alojamiento con un toque cultural con tres objetivos como la mejora en el aspecto decorativo, promocionar a los artistas, y un medio de venta de sus obras actuando de intermediario.

\subsection{Centro de degustación enogastronómica}

Los productos agroalimentarios son un recurso económico y turístico muy importante en el medio rural. De esta forma, a través de la transformación de un producto agrario, en un bien de consumo, se está consiguiendo diversificar la economía del medio rural basada, hasta hace pocas décadas, casi en exclusiva en el sector agrario. La obtención de una figura de calidad puede repercutir positivamente en un mayor beneficio económico y prestigio social de ese producto.

Este centro pretende realizar, con carácter regular, una serie de actividades programadas en relación a catas y degustaciones de vino de la D.O. Cigales, pero además de otros productos elaborados en los diferentes municipios como los quesos, morcillas, el pan, repostería, y otros productos que contribuirían a un reconocimiento mayor del proceso de valoración y de la obtención de la materia prima.

Aparte de los productos propios de los municipios, también se pretende que los empresarios sean las personas encargadas de explicar y organizar estas actividades, como forma de diversificación económica de sus negocios, y de ofrecer un producto turís- 
tico diferente al visitante, en auge desde hace tiempo debido al interés que suscita el enoturismo en los municipios vinculados a la Denominación de Origen.

A la hora de buscar financiación para poder hacer realidad los proyectos propuestos, existen varios organismos donde se pueden solicitar ayudas, de forma directa o indirecta, tanto para las bodegas en sí, como para su entorno, protegiendo el paisaje cultural. Destacar la Administración Local, la Administración Provincial ${ }^{16}$, la Junta de Castilla y León ${ }^{17}$, las Administraciones Nacionales ${ }^{18}$ y la Unión Europea ${ }^{19}$, gestionando una parte de estas ayudas a través de los Grupos de Acción Local del programa LEADERCAL ${ }^{20}$.

Estas líneas de ayudas pueden sufrir variaciones debido a la actual situación en la que las ayudas o subvenciones se modifican o suprimen. Además de la iniciativa pública existe también la privada, bien de empresas, que estén interesados en la participación de los proyectos, o bien de diversas Fundaciones cuyos objetivos se enmarquen en los proyectos a realizar.

Aparte de estas formas de financiación, también en algunos proyectos propuestos se puede trabajar mediante Campos de Trabajo y/o Asociaciones que pueden aportar a las propuestas financiación "en especie" para aminorar los gastos. Particularmente, el Campo de trabajo se puede utilizar para la recuperación del patrimonio arquitectónico y la naturaleza ${ }^{21}$.

Por otro lado, mientras no se tenga la catalogación como BIC no se puede optar a ninguna ayuda directa ni regional ni estatal para rehabilitar o restaurar el patrimonio. Es por ello muy importante que se consiga que las bodegas tengan la protección para poder aspirar a este tipo de ayudas, tanto la del Instituto del Patrimonio de España, como la del $1 \%$ cultural.

16. Plan Provincial de Obras y Servicios, Fondo Cooperación Local, Subvenciones Ayuntamiento-Diputación, Subvenciones Gastos mantenimiento, Plan Provincial de Cooperación.

17. Talleres de Empleo y/o Escuelas Taller, Fondo de Cooperación Local, Subvenciones de Infraestructuras Turísticas, Dirección General de Patrimonio, Consejería de Economía (Agencia de Desarrollo Económico).

18. Ministerio de Agricultura, Alimentación y Medio Ambiente, Ministerio de Educación, Cultura y Deporte (El 1\% cultural), Instituto de Patrimonio de España.

19. FEOGA: Fondo Europeo de Orientación y Garantía Agrícola, Fondo Social Europeo, Fondo de cohesión, Programas Cultura, Programa Europa con los Ciudadanos 2007-2013, Programa Europa Creativa 20142020 y ERASMUS+.

20. Se distribuyen en tres Grupos de Acción Local: 1- Proder Zona Centro que se ubican Mucientes, Corcos del Valle, Trigueros del Valle, Quintanilla de Trigueros y Cubillas de Santa Marta. 2- Adri-Cerrato Palentino, en el que se integra Dueñas y 3- Duero-Esgueva que están Valoria la Buena y San Martín de Valvení. Las localidades de Cigales, Cabezón de Pisuerga y Fuensaldaña, están excluidas de los programas de desarrollo al considerarse alfoz de Valladolid y su índice de ruralidad es muy bajo, por lo que se consideran suficientemente desarrollados y no necesitan este tipo de ayudas.

21. Se puede obtener más información sobre Campos de Trabajo en www.campodetrabajo.com 
Estas acciones se pueden desarrollar en fases o reduciendo costes según la forma de gestionarlo. Las intervenciones se han calculado en base estimada al acondicionamiento general del inmueble para las acciones que se plantean. Claro está que cuanta más complejidad requiera el proyecto, mayor encarecimiento del mismo, por lo que nos hemos ido a unas situaciones de tipo medio para poder desarrollar de forma eficiente los proyectos.

El presupuesto utilizado para calcular el coste de las diversas actuaciones es estimado en base a precio mercado y optimizando recursos, puesto que de las intervenciones que se proponen pueden tener mayor o menor coste económico según la complejidad de la intervención. Es por ello que el coste propuesto está ajustado a unos mínimos con garantía de éxito ${ }^{22}$.

Por consiguiente, el listado de intervenciones con el presupuesto estimado según las acciones es el siguiente.

Cuadro 3. Presupuestos estimados de las propuestas de intervención

\begin{tabular}{|l|l|}
\hline Intervenciones & \\
\hline 1. Ecomuseo & $42.100 €$ \\
2. Museo al Aire Libre & $90.100 €$ \\
3. Festival de Bodegas tradicionales & Desde $12.900 €$ \\
4. Sala de Exposiciones & $177.700 €$ \\
5. Vivero de empresas creativo-culturales & $463.100 €$ \\
6. Bodegas de creación & $767.000 €$ \\
7. Restaurante cultural & $272.000 €$ \\
8. Alojamiento rural cultural & $419.000 €$ \\
9. Centro de degustación enogastronómica & $189.900 €$ \\
\hline
\end{tabular}

Fuente: Elaboración propia.

22. Debido a la diversidad de propuestas que se plantean, un conjunto de nueve, resulta inabarcable en este trabajo detallar de forma individual y detallada todos los costes derivados de las diferentes iniciativas. Por este motivo se señalan algunos de los factores comunes a todas las propuestas destacando la redacción y el visado de proyectos técnicos, la elaboración de proyectos museográficos, gastos en promoción, adquisición de materiales, etc. Por resaltar un ejemplo en concreto se va a especificar el caso del Museo al aire libre. En este caso, además de los aspectos citados anteriormente hay que destacar algunos específicos como el adecentamiento de las laderas, la contratación de artistas, gastos en la señalización, etc. 


\section{Conclusión}

Las localidades vinculadas a la D.O. Cigales poseen un patrimonio destacado y una cultura del vino muy arraigada entre sus habitantes. Aparte, los barrios de bodegas son una seña de identidad de las personas que habitan estos espacios, ya que esta actividad económica se lleva desarrollando, con mayor o menor intensidad en este territorio durante siglos. Partiendo de esto, no es de extrañar las posibilidades de desarrollo económico, turístico y cultural que posee el patrimonio relacionado con el vino, como hemos ido desgranando a lo largo del artículo.

Es fundamental concienciar a la propia sociedad del potencial presente en su territorio y que valoren lo existente para poder trabajar en futuras acciones concretas. Si la sociedad no se preocupa de mantener lo que han heredado, es muy difícil legarlo a los descendientes en condiciones dignas, desaprovechando unas oportunidades manifiestas para trabajar en su desarrollo.

El principal enemigo es el abandono y desuso de las bodegas. El patrimonio requiere de unas condiciones mínimas de mantenimiento para evitar la dejadez que produce el deterioro, el derrumbe y la pérdida irremediable de estos bienes, por lo que es necesario tenerlo abierto y ventilado para que siga en pie. Estamos hablando de un patrimonio con cientos de años en la mayor parte de los casos, con unas características propias, que confieren a este espacio, un atractivo capaz de mover a personas interesadas en este mundo, así como a los turistas que recorren estos campos del centro de Castilla y León.

Asimismo, hemos propuesto diversas acciones poniendo de manifiesto la capacidad real de generar actividades entorno a estas bodegas dotándolas de un uso diferente a los tradicionales. Es necesario que exista una buena gestión para recuperar estos espacios y hacerlos atractivos a los visitantes, y que el conjunto de los municipios que conforman la D.O. Cigales y su población, se sientan orgullosos e identificados con su patrimonio y su cultura.

Habiendo interés en afrontar un proyecto, la financiación se puede acometer. Con mayor o menor ritmo de ejecución, con implicaciones públicas o privadas, con proyectos más simples o complejos. Con estas premisas el trayecto está más claro si existe la apuesta decidida por parte de los agentes responsables de llevar a cabo estas iniciativas, que permitirían preservar el patrimonio etnográfico de las bodegas, así como diversificar el tejido económico de estas localidades creando nuevos puestos de trabajo que ayudarían a revitalizar estás localidades, algunas de las cuales, llevan en una espiral de retroceso social y económico desde mediados del siglo XX. 


\section{Bibliografía}

Alonso Santos, J.L. (2003) Redes y procesos de innovación en las comarcas vinícolas de Castilla y León: el ejemplo de la D.O. Bierzo. Boletín de la Asociación de Geógrafos Españoles, 36, $43-60$.

Alonso Santos, J.L. et al. (2003) Los espacios vitivinícolas en Castilla y León: la evolución hacia un sistema productivo de calidad. Boletín de la Asociación de Geógrafos Españoles, 35, 101-122.

Aparicio, J. et al. (2008) La Ribera del Duero, geografía de un medio innovador en torno a la viticultura. Scripta Nova, 277.

Barbieri, N. (2011) Nuevas políticas, nuevas miradas y metodologías de evaluación ¿Cómo evaluar el retorno social de las políticas culturales?. Papiers, 96/2, 489.

Camarero Bullón, C. (1989) La filoxera en la provincia de Burgos. Estudios Geográficos, 197, 531-552.

Carricajo Carbajo, C. (2011) 50+1 construcciones vernáculas. Valladolid, Diputación de Valladolid.

De Luis Calabuig, E. (2012) Actas del Simposio sobre Patrimonio cultural, paisaje e infraestructuras. Valladolid, Fundación del Patrimonio histórico de Castilla y León.

Duque Herrero, C. (2006) Vino, lagares y bodega. Bodega-Aula de Interpretación. Mucientes, Ayuntamiento de Mucientes.

Fernández Portela, J. (2012) Cambios en la industria y en el paisaje vitivinícola de la Denominación de Origen de Cigales (Castilla y León, España). Estudios Geográficos, 272, 63-90.

Fernández Portela, J. \& García Isla, V. (2012) La atracción del vino en los viajeros europeos de los siglos XVIII y XIX. Polígonos, Revista de Geografía, 23, 235-262.

Figueroa y Melgar, A. (1971) Viajes románticos por España. Madrid, Escuelas Profesionales Sagrado Corazón.

Franco Jubete, F. \& Luis del Rio, S. (2005) Cultura vitivinícola del Cerrato castellano. Viñedos, Vinos y Bodegas del Cerrato palentino. Palencia, Itagra.

Huetz de Lems, A. (1967) Vignobles et vins du nord-ouest de l'espagne. Bordeaux, Institut de Géographie, Faculté des lettres.

Huetz de Lemps, A. (2005) Vinos y Viñedos de Castilla y León. Valladolid, Consejería de Agricultura y Ganadería de la Junta de Castilla y León.

Gómez Lacort, J.E. et al. (1998) Las bodegas. El acento de un paisaje y su contenido en Cubillas de Santa Marta. Valladolid, Fundación etnográfica Joaquín Díaz, Castilla Ediciones.

Jové Sandoval, F \& Sáinz Guerra, J.L. (coords) (2011) Construcción con tierra. Tecnología y arquitectura. Congresos de Arquitectura de tierra en Cuenca de Campos 2010/11. Valladolid, E.T.S. Arquitectura de Valladolid.

Marino Pascual, J. (2010) Los Barrios de Bodegas: El ser de La Rioja. Logroño, Quel.

Piqueras Haba, P. (2005) La filoxera en España y su difusión espacial: 1878-1926. Cuadernos de Geografia, 77, 101-136.

Plasencia, P. (1995) Los vinos de España vistos por los viajeros europeos. Madrid, Ministerio de Agricultura, Pesca y Alimentación. 
Sánchez del Barrio, A. \& Carricajo Carbajo, C. (1995) Arquitectura popular. Valladolid, Editorial Castilla.

Sánchez Hernández, J.L. (2002) La renovación de las bases productivas en la industria vinícola de Rueda, en Méndez, R. y Alonso, J.L. (eds.), Sistemas locales de empresas y redes de innovación en Castilla La Mancha y Castilla y León. Ediciones Universidad de Salamanca. Salamanca, p, 231-257

Sánchez Hernández, J.L. (2003) Capital exógeno y procesos de innovación en la industria vinícola de la Denominación de Origen "Toro". Boletín de la Asociación de Geógrafos Españoles, 36, 61-79.

Solano Sobrado, M.T. (1991) La crisis del viñedo. La filoxera en España. Tesis Doctoral. Madrid, Editorial de la Universidad Complutense de Madrid.

Teste, L. (1959) Viaje por España. Valencia, Castalia.

Valdeón Baruque, J. (2006) La Reconquista: el concepto de España: unidad y diversidad. Madrid, Espasa-Calpe. 